\title{
Misclassification of Plasmodium infections by conventional microscopy and the impact of remedial training on the proficiency of laboratory technicians in species identification
}

\author{
Peter Obare ${ }^{1 *}$, Bernhards Ogutu ${ }^{1,4}$, Mohammed Adams $^{2}$, James Sande Odera ${ }^{1}$, Ken Lilley $^{3}$, David Dosoo ${ }^{2}$,
} Christine Adhiambo ${ }^{1}$, Seth Owusu-Agyei ${ }^{2}$, Fred Binka ${ }^{4}$, Elizabeth Wanja ${ }^{1}$ and Jacob Johnson ${ }^{1}$

\begin{abstract}
Background: Malaria diagnosis is largely dependent on the demonstration of parasites in stained blood films by conventional microscopy. Accurate identification of the infecting Plasmodium species relies on detailed examination of parasite morphological characteristics, such as size, shape, pigment granules, besides the size and shape of the parasitized red blood cells and presence of cell inclusions. This work explores misclassifications of four Plasmodium species by conventional microscopy relative to the proficiency of microscopists and morphological characteristics of the parasites on Giemsa-stained blood films.

Case description: Ten-day malaria microscopy remedial courses on parasite detection, species identification and parasite counting were conducted for public health and research laboratory personnel. Proficiency in species identification was assessed at the start (pre) and the end (post) of each course using known blood films of Plasmodium falciparum, Plasmodium malariae, Plasmodium ovale and Plasmodium vivax infections with densities ranging from 1,000 to 30,000 parasites/ $\mu \mathrm{L}$. Outcomes were categorized as false negative, positive without speciation, P. falciparum, P. malariae, P. ovale, P. vivax and mixed infections.
\end{abstract}

Discussion and evaluation: Reported findings are based on 1,878 P. falciparum, 483 P. malariae, 581 P. ovale and 438 P. vivax cumulative results collated from 2008 to 2010 remedial courses. Pre-training false negative and positive misclassifications without speciation were significantly lower on $P$. falciparum infections compared to non-falciparum infections $(p<0.0001)$. Post-training misclassifications decreased significantly compared to pre- training misclassifications which in turn led to significant improvements in the identification of the four species. However, $P$. falciparum infections were highly misclassified as mixed infections, $P$. ovale misclassified as $P$. vivax and $P$. vivax similarly misclassified as $P$. ovale $(p<0.05)$.

Conclusion: These findings suggest that the misclassification of malaria species could be a common occurrence especially where non-falciparum infections are involved due to lack of requisite skills in microscopic diagnosis and variations in morphological characteristics within and between Plasmodium species. Remedial training might improve reliability of conventional light microscopy with respect to differentiation of Plasmodium infections.

Keywords: Microscopy, Species, Morphology, Misclassification, Training

\footnotetext{
* Correspondence: peter.obare@usamru-k.org

${ }^{1}$ Kenya Medical Research Institute/United States Army Medical Research Unit,

Kenya, Malaria Diagnostics Centre, Box 54 - 40100, Kisumu, Kenya

Full list of author information is available at the end of the article
} 


\section{Background}

Malaria diagnosis in most settings is largely dependent on the demonstration of parasites in stained blood films by conventional microscopy. This enables parasite detection, identification of Plasmodium species and estimation of parasite densities. Accurate identification of the infecting Plasmodium species relies on detailed examination of parasite morphological characteristics such as size, shape, pigmentation, besides the size and shape of the parasitized red blood cells and the inclusions therein $[1,2]$. Medical text books, reference literature and atlases that describe morphological features of Plasmodium species already exist to aid microscopic diagnosis of malaria infections [1].

Plasmodium species traditionally associated with human infections are Plasmodium falciparum, Plasmodium vivax, Plasmodium malariae and Plasmodium ovale. However, Plasmodium knowlesi associated with long tailed macaque monkeys has of late crossed over to humans and is now considered by some to be the fifth human malaria $[3,4]$. Since Plasmodium species differ with respect to their biology, clinical symptoms, and treatment requirements [5,6]; accurate identification of Plasmodium infections is of critical importance with regard to formulation and deployment of effective intervention strategies in endemic regions [6].

Plasmodium falciparum is the most prevalent malaria infection in Africa and accounts for the largest proportion of clinical cases $[7,8]$. Plasmodium malariae infections are frequently found in sympatry with $P$. falciparum infections [7,9-11], but cases go undetected unless molecular methods are used for diagnosis [10]. The burden of $P$. ovale is thought to be highest in sub-Saharan Africa. However, its prevalence is deemed to be lower compared to $P$. malariae $[11,12]$. The near absence of Duffy positive phenotype in the local populations seems to exclude transmission of $P$. vivax in sub-Saharan Africa [13,14] except in countries such as Djibouti, Eritrea, Ethiopia, Somalia, and Sudan [15].

This work explores misclassification of four Plasmodium species by conventional microscopy relative to the proficiency of microscopists and morphological characteristics of the parasites on Giemsa stained blood films.

\section{Case description \\ Methods}

\section{Remedial microscopy courses}

Ten-day remedial malaria microscopy courses were conducted by Kenya Medical Research Institute/US Army Medical Research Unit-Kenya (KEMRI/USAMRU-K), Malaria Diagnostics Centre (MDC) and Kintampo Health Research Center (KHRC), Ghana from 2008 to 2010 as part of a multilateral capacity building strategy for laboratory personnel in public health and research institutions.
Training sessions had both theory-based and practice-based components on parasite detection (presence or absence), morphological characterization of Plasmodium species, and parasite counting techniques. The practice-based component focused on examination of reference malaria slide sets under the guidance of expert microscopists.

\section{Malaria slide sets}

Slide sets used for training and proficiency assessments consisted of $P$. falciparum, $P$. malariae, $P$. ovale and $P$. vivax infections with parasite densities ranging from 1,000 - 30,000 parasites/ $\mu \mathrm{L}$. Each slide had a thick and thin film stained using buffered 3\% Giemsa solution (Sigma-Aldrich GS1L-1 L) for 60 minutes and integrity of the films maintained with microscope cover-glasses (Fisher Scientific 12-543-D) and DPX mountant (Polysciences 13512). Parasitological validation was conducted via multiple independent examinations of coded sets by expert microscopists in addition to Plasmodium genus and species-specific PCR assays.

\section{Proficiency assessments}

Assessments in species identification were by way of pre and post-training examination of 20 slides sets with the above mentioned species distributed equally between P. falciparum and non-falciparum infections. Pre and posttraining test sets were coded differently and participants allowed five minutes to examine each slide and report their findings. Results were evaluated, feedback provided, and hands-on revision exercises conducted under the guidance of expert microscopists.

\section{Data analysis}

1,878 P. falciparum, 483 P. malariae, 581 P. ovale and 438 $P$. vivax cumulative results were collated and outcomes per species categorized as false negative (FN), positive without speciation (PS), P. falciparum, P. malariae $P$. ovale, $P$. vivax and mixed infections (MX). Findings per category were converted to proportions and differences within and between Plasmodium infections analysed using Marascuilo Chi square test for multiple proportions with post hoc comparisons. Significant differences within species were $X^{2}$ values $\geq 12.59$, df $6, \mathrm{p}<0.05$ and between species $X^{2}$ values $\geq 7.82$, df $3, \mathrm{p}<0.05$ [16].

\section{Results}

\section{Misclassifications on $P$. falciparum infections}

Pre-training misclassification of $P$. falciparum infections as PS was significantly higher compared to misclassification as FN, misclassification as $P$. ovale, misclassification as P. vivax in addition to misclassification as MX ( $\mathrm{p}<0.0001)$. Misclassification as $P$. malariae was significantly higher compared to misclassification as FN together with misclassification as $P$. ovale $(\mathrm{p}<0.005)$ while misclassification 
as MX was significantly higher compared to misclassification as $P$. ovale $(\mathrm{p}=0.029)$. There were significant reductions in post-training misclassifications except for residual misclassification as $P$. ovale which was marginally lower and residual misclassification as MX which became marginally higher compared to pre-training results $(\mathrm{p}>0.05)$. Residual misclassification as PS was significantly lower compared to residual misclassification as $P$. ovale, residual misclassification as $P$. vivax besides residual misclassification as MX ( $\mathrm{p}<0.005)$. Reductions post-training misclassifications significantly improved accurate identification of $P$. falciparum infections compared to pre-training results (Figure 1 \& Additional files 1, 2, 3).

\section{Misclassifications on $P$. malariae infections}

Pre-training misclassification of $P$. malariae infections as FN was significantly higher compared to all the other misclassifications $(\mathrm{p}<0.05)$, misclassification as $\mathrm{MX}$ significantly lower compared to the other remaining misclassifications $(\mathrm{p}<0.0001)$ while misclassification as PS was significantly higher compared to misclassification as $P$. $\operatorname{vivax}(\mathrm{p}=0.012)$. There were significant reductions in post-training misclassifications except for residual misclassification as $P$. ovale which was marginally lower along with residual misclassification as MX which became significantly higher compared to pretraining results $(\mathrm{p}<0.0001)$. Residual misclassification as PS was significantly lower compared to other residual misclassifications $(\mathrm{p}<0.05)$ except residual misclassification as FN. Reductions in post-training misclassifications resulted in a significant improvement in accurate identification of $P$. malariae infections (Figure 1 \& Additional files 1, 2, 3).

\section{Misclassifications on $P$. ovale infections}

Pre-training misclassification of $P$. ovale infections as MX was significantly lower compared to all the other misclassifications $(\mathrm{p}<0.05)$, misclassification as FN significantly higher compared to misclassification as $P$. falciparum together with misclassification as $P$. vivax $(\mathrm{p}<0.005)$ whereas misclassification as PS was higher than misclassification as $P$. falciparum $(\mathrm{p}=0.004)$. There were significant reductions in post-training misclassifications except for residual misclassification as $P$. vivax which became marginally higher and residual misclassification as $\mathrm{MX}$ which became significantly higher compared to pre-training results. Notably, residual misclassification of $P$. ovale infections as $P$. vivax became the most common misclassification compared to other residual misclassifications $(\mathrm{p}<0.05)$. This was followed by misclassification as $P$. malariae which became significantly higher than residual misclassification as FN besides to residual misclassification as PS $(p<0.05)$. The observed reductions in some of the post-training misclassifications contributed to a significant improvement in accurate identification of $P$. ovale infections (Figure 1 \& Additional files 1, 2, 3).

\section{Misclassifications on $P$. vivax infections}

Pre-training misclassification of $P$. vivax infections as MX was significantly lower compared to all the other misclassifications $(\mathrm{p}<0.0001)$ whereas misclassification as FN was significantly higher compared to misclassification as $P$. falciparum together with misclassification as $P$. malariae $(\mathrm{p}<0.05)$. There were significant reductions in post-training misclassifications except for residual misclassifications as $P$. ovale along with residual

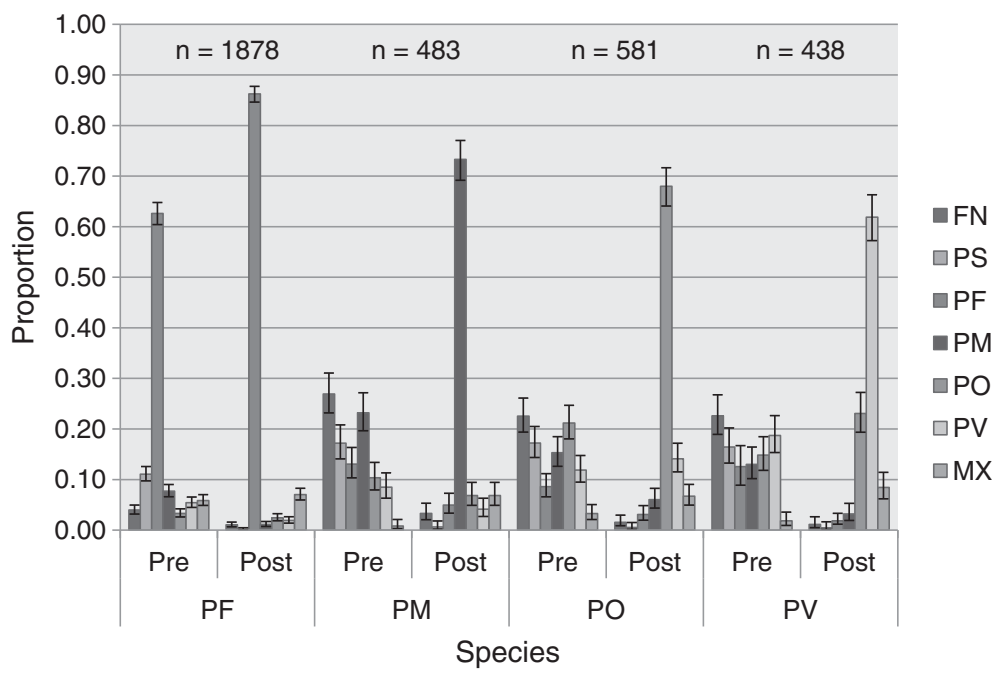

Figure 1 Cumulative distribution of outcomes across Plasmodium species. Note: 95\% Cls constructed using Wilson's procedure without continuity correction. 
misclassification as MX which became significantly higher compared to pre-training results. Post-training misclassification as $P$. ovale was the most common residual error $(\mathrm{p}<0.0001)$ followed with residual MX when compared to the rest of the residual misclassifications $(\mathrm{p}<0.005)$ except residual misclassification as $P$. malariae. The reductions in post-training misclassifications resulted in a significant improvement in accurate identification of $P$. vivax infections (Figure 1 \& Additional files 1, 2, 3).

\section{Misclassifications between Plasmodium infections}

Pre-training misclassification of $P$. falciparum infections as FN along with misclassification as PS were significantly lower compared to similar misclassifications on non-falciparum infections $(\mathrm{p}<0.05)$. Misclassification of $P$. falciparum infections as $P$. malariae was equally lower compared to similar misclassification on $P$. ovale and $P$. vivax infections $(\mathrm{p}<0.05)$ and misclassification of $P$. falciparum infections as $P$. ovale significantly lower compared to same misclassification on $P$. malariae and $P$. vivax infections $(\mathrm{p}<0.001)$. Misclassification of $P$. falciparum infections as $P$. vivax was significantly lower compared to equivalent misclassification on $P$. ovale infections $(\mathrm{p}<0.001)$. Misclassification of $P$. falciparum infections as MX was significantly higher compared to similar misclassification of $P$. malariae, $P$. ovale and $P$. vivax infections $(\mathrm{p}<0.05)$. Residual post-training misclassification of $P$. falciparum infections as $P$. malariae was significantly lower compared to equivalent misclassification on $P$. ovale infections $(\mathrm{p}<0.001)$. Misclassification of $P$. ovale infections as $P$. vivax was significantly higher compared to similar misclassifications on $P$. falciparum and $P$. malariae infections respectively $(\mathrm{p}<0.001)$ and misclassification of $P$. vivax infections as $P$. ovale equally higher compared to equivalent misclassification on $P$. falciparum and $P$. malariae infections $(\mathrm{p}<0.001)$ (Figure 1 \& Additional file 4).

\section{Discussion and evaluation}

Publications by different groups have shown that laboratory personnel involved in microscopic diagnosis of malaria experience difficulties in differentiating Plasmodium infections [17-19]. Those observations are consistent with results presented here with respect to inaccuracies associated with microscopic identification of non-falciparum infections. Higher pre-training false negative and positive results without speciation on nonfalciparum infections suggest most of the participants were not familiar with non-falciparum infections and, therefore, opted to report such infections as negative or positive without indicating the appropriate species. Familiarity with non-falciparum during post- training assessments reduced misclassifications within and between species considerably compared to pretraining results.

Lack of significant trends in pre-training misclassification of the four Plasmodium infections based on species outcomes were most probably a result of guess work. Nonetheless, significant trends in misclassifications emerged in post-training assessments despite there being significant reductions in species identification errors. These shifts were majorly misclassification of $P$. falciparum infections as mixed infections, misclassification of $P$. ovale infections as $P$. vivax and misclassification of $P$. vivax infections as P. ovale.

Misclassification of $P$. falciparum infections as mixed infections can be attributed to morphological variations in parasites forms. Whereas early trophozoites stages are smaller in size and have tiny nucleus and delicate cytoplasm, mature stages are relatively bigger in size, more compact and pigmented. This might have led some participants to believe non-falciparum species were also present on some of the blood films. Misclassification of $P$. ovale infections as $P$. vivax and vice versa might have been due to similarities in morphological characteristics such as cell inclusions i.e. James' and Schüffner's clefts, host cell enlargement and rounded gametocytes. These overlaps in morphological characteristics make differentiation of the two species extremely challenging even for experienced microscopists. A key feature commonly used in differentiating these two species is the presence of distinct amoeboid or fragmented forms and marked enlargement of the host cell in P. vivax infections.

Other than possession of requisite skills in morphological identification, thin films are equally important in enhancing the identification of Plasmodium species. Most of the pre-training misclassifications were as a result of thick film examination. While thick films are useful in concentrating parasites for easy identification; the presence of multiple layers of red blood cells which are subsequently de-haemoglobinized during staining distorts essential morphological features needed for species differentiation. Unlike thick films, single layers of red blood cells at the tail of thin films and fixation in alcohol before staining helps in preserving species specific diagnostic details such as red blood cell shape and size, parasite morphology, pigment color and presence of cell inclusions which would otherwise be lost during thick film staining. Therefore, thin films are more reliable in species differentiation compared to thick films. Ideally, thick films should be used for presumptive identification of parasites and estimation of parasite counts whereas thin films should be used for definitive identification of species.

Post-training results did show significant reductions in misclassifications despite the residual errors reported. 
Occurrence of residual errors can be attributed to variations in morphological characteristics in various stages of parasite development within species and overlap in morphological characteristics between species. It is also important to note that proficiency in microscopic diagnosis of malaria is built over time, based on frequent exposure to different Plasmodium infections and their respective multiple morphological characteristics.

\section{Conclusion}

Conventional light microscopy despite being the "gold standard" for malaria diagnosis is wrought with many challenges. These findings suggest misclassification of Plasmodium infections may be a common occurrence where non-falciparum species are involved. This can be attributed to lack of requisite skills in morphological characterization on the part of microscopists as well as morphological variations in parasite characteristics within and between species. Improving the skill sets of malaria microscopists through remedial training may reduce species misclassification errors associated with microscopy and ensure reliability of patient care and research results. Independent confirmation of species by expert microscopy in addition to molecular analysis of specimens in both patient care setting and research studies is highly recommended.

\section{Additional files}

Additional file 1: FN misclassifications within Plasmodium

infections. Note: Values represent differences between comparisons together with the corresponding $X 2$ statistic, $X$ indicates redundant comparisons while + indicates significant differences.

Additional file 2: PS misclassifications within Plasmodium infections. Note: Values represent differences between comparisons together with the corresponding X2 statistic, $\mathrm{X}$ indicates redundant comparisons while + indicates significant differences.

Additional file 3: Species misclassifications within Plasmodium infections. Note: Values represent differences between comparisons together with the corresponding $X 2$ statistic, $X$ indicates redundant comparisons while + indicates significant differences.

Additional file 4: Misclassifications between Plasmodium infections. Note: Values represent differences between comparisons together with the corresponding $\mathrm{X} 2$ statistic, $X$ indicates redundant comparisons while + indicates significant differences.

\section{Abbreviations}

FN: False negative; PS: Positive without speciation; PF: Plasmodium falciparum; PM: Plasmodium malariae; PO: Plasmodium ovale; PV: Plasmodium vivax; MX: Mixed infections.

\section{Competing interests}

All authors declared that they have no competing interest.

\section{Authors' contributions}

PO designed the course, taught the courses, analyzed the data and drafted the manuscript. BO designed the course, facilitated financial and logistical support for courses conducted in Kenya and Ghana and assisted in drafting the manuscript. MA and JSO were the senior instructors of the courses. KL critiqued training methodology and results. CA designed the course, was responsible for logistics and taught the courses. DO was responsible for logistics and taught the courses. SO and FB facilitated financial and logistical support for courses conducted in Ghana. EW and JJ facilitated financial and logistical support for courses conducted in Kenya and assisted in drafting the manuscript. All authors read and approved the final manuscript.

\section{Acknowledgements}

Remedial courses were conducted with funds from US Department of Defense Global Emerging Infections Surveillance System (GEIS), US President's Malaria Initiative (PMI) and Malaria Clinical Trials Alliance (MCTA). Plasmodium vivax blood films were obtained from Armed Forces Research Institute of Medical Sciences (AFRIMS) and Malaria Research Reference Reagent Resource Center (MR4). We thank Kingsley Kayan, Dominic Dery of KHRC and Cornel Arima, Agneta Ogolo, Cephas Oyieke, Michael Ayaya, Catherine Sumbi, Everlyne Omondi of MDC for technical assistance during the workshops, Emmanuel Mahama of KHRC and Reuben Oriko of MDC for data management. This manuscript is published with the approval of Director, KEMRI.

\section{Author details}

'Kenya Medical Research Institute/United States Army Medical Research Unit, Kenya, Malaria Diagnostics Centre, Box 54 - 40100, Kisumu, Kenya. ${ }^{2}$ Kintampo Health Research Center, Box 200, Kintampo, Brong Ahafo Region, Ghana.

${ }^{3}$ Australian Army Malaria Institute, Weary Dunlop Drive, Enoggera, QLD 4051, Australia. ${ }^{4}$ Malaria Clinical Trials Alliance, INDEPTH-Network, Box 213, Kanda, Accra, Ghana.

Received: 14 January 2013 Accepted: 22 March 2013

Published: 27 March 2013

\section{References}

1. Lee KS, Cox-Singh J, Singh B: Morphological features and differential counts of Plasmodium knowlesi parasites in naturally acquired human infections. Malar J 2009, 8:73.

2. Wongsrichanalai C, Barcus MJ, Muth S, Sutamihardja A, Wernsdorfer WH: A review of malaria diagnostic tools: microscopy and rapid diagnostic test (RDT). AmJTrop Med Hyg 2007, 77:119-127.

3. Lee KS, Divis PC, Zakaria SK, Matusop A, Julin RA, Conway DJ, Cox-Singh J, Singh B: Plasmodium knowlesi: reservoir hosts and tracking the emergence in humans and macaques. PLoS Pathog 2011, 7:e1002015.

4. Kantele A, Jokiranta TS: Review of cases with the emerging fifth human malaria parasite, Plasmodium knowlesi. Clin Infect Dis 2011, 52:1356-1362.

5. Mehlotra RK, Lorry K, Kastens W, Miller SM, Alpers MP, Bockarie M, Kazura JW, Zimmerman PA: Random distribution of mixed species malaria infections in Papua New Guinea. AmJTrop Med Hyg 2000, 62:225-231.

6. Scopel KK, Fontes CJ, Nunes ÁC, Horta MF, Braga ÉM: High prevalence of Plasmodium malariae infections in a Brazilian Amazon endemic area (Apiacás-Mato Grosso State) as detected by polymerase chain reaction. Acta Trop 2004, 90:61-64

7. Bruce MC, Macheso A, McConnachie A, Molyneux ME: Comparative population structure of Plasmodium malariae and Plasmodium falciparum under different transmission settings in Malawi. Malar J 2011, 10:38.

8. Hay SI, Okiro EA, Gething PW, Patil AP, Tatem AJ, Guerra CA, Snow RW: Estimating the global clinical burden of Plasmodium falciparum malaria in 2007. PLoS Med 2010, 7:e1000290.

9. Bousema JT, Drakeley CJ, Mens PF, Arens T, Houben R, Omar SA, Gouagna LC, Schallig H, Sauerwein RW: Increased Plasmodium falciparum gametocyte production in mixed infections with P. malariae. AmJTrop Med Hyg 2008, 78:442-448.

10. Collins WE, Jeffery GM: Plasmodium malariae: parasite and disease. Clin Microbiol Rev 2007, 20:579-592.

11. Mueller I, Zimmerman PA, Reeder JC: Plasmodium malariae and Plasmodium ovale: the "bashful" malaria parasites. Trends Parasitol 2007, 23:278-283.

12. Sutherland CJ, Tanomsing N, Nolder D, Oguike M, Jennison C, Pukrittayakamee S, Dolecek C, Hien TT, Do Rosario VE, Arez AP, Pinto J, Michon P, Escalante AA, Nosten F, Burke M, Lee R, Blaze M, Otto TD, Barnwell JW, Pain A, Williams J, White NJ, Day NP, Snounou G, Lockhart PJ, Chiodini PL, Imwong M, Polley SD: Two nonrecombining sympatric forms of the human malaria parasite Plasmodium ovale occur globally. I Infect Dis 2010, 201:1544-1550 
13. Mueller I, Galinski MR, Baird JK, Carlton JM, Kochar DK, Alonso PL, del Portillo HA: Key gaps in the knowledge of Plasmodium vivax, a neglected human malaria parasite. Lancet Infect Dis 2009, 9:555-566.

14. Culleton RL, Mita T, Ndounga M, Unger H, Cravo PV, Paganotti GM, Takahashi N, Kaneko A, Eto H, Tinto H, Karema C, D'Alessandro U, Do Rosário V, Kobayakawa T, Ntoumi F, Carter R, Tanabe K: Failure to detect Plasmodium vivax in West and Central Africa by PCR species typing. Malar J 2008, 7:174.

15. Snow RW, Omumbo JA: Malaria. In Disease and Mortality in Sub-Saharan Africa. 2nd edition. Edited by Jamison DT, Feachem RG, Makgoba MW, Bos ER, Baingana FK, Hofman KJ, Rogo KO. Washington (DC): World Bank; 2006. Chapter 14. http://www.ncbi.nlm.nih.gov/books/NBK2286/.

16. Multiple proportions and post hoc analysis. http://www.statstodo.com/ MultiProp_Pgm.php.

17. Rosas-Aguirre Á, Gamboa D, Rodriguez H, Llanos-Zavalaga F, Aguirre K, Llanos-Cuentas A: Use of standardized blood smear slide sets for competency assessment in the malaria microscopic diagnosis in the Peruvian Amazon. Rev Peru Med Exp Salud Publica 2010, 27:540-547.

18. Edson DC, Glick T, Massey LD: Detection and identification of malaria parasites: a review of proficiency test results and laboratory practices. Lab Med 2010, 4:719-723.

19. Barber BE, William T, Grigg MJ, Yeo TW, Anstey NM: Limitations of microscopy to differentiate Plasmodium species in a region co-endemic for Plasmodium falciparum. Plasmodium vivax and Plasmodium knowlesi. Malar J 2013, 12:8.

doi:10.1186/1475-2875-12-113

Cite this article as: Obare et al:: Misclassification of Plasmodium infections by conventional microscopy and the impact of remedial training on the proficiency of laboratory technicians in species identification. Malaria Journal 2013 12:113.

\section{Submit your next manuscript to BioMed Central and take full advantage of:}

- Convenient online submission

- Thorough peer review

- No space constraints or color figure charges

- Immediate publication on acceptance

- Inclusion in PubMed, CAS, Scopus and Google Scholar

- Research which is freely available for redistribution 